\title{
Influencia de la Extensibilidad Isquiosural en la Morfología Sagital del Raquis e Inclinación Pélvica en Deportistas
}

\author{
Influence of Hamstring Extensibility on Sagittal \\ Spinal Curvatures and Pelvic Inclination in Athletes \\ "José María Muyor; **Fernando Alacid; ***Pedro L. Rodríguez-García \& *** Pedro A. López-Miñarro
}

MUYOR, J. M.; ALACID, F.; RODRÍGUEZ-GARCÍA, P. L. \& LÓPEZ-MIÑ̃ARO, P. A. Influencia de la extensibilidad isquiosural en la morfología sagital del raquis e inclinación pélvica en deportistas. Int. J. Morphol., 30(1):176-181, 2012.

RESUMEN: El objetivo de este estudio fue determinar la influencia de la extensibilidad isquiosural en la morfología sagital del raquis e inclinación pélvica en deportistas jóvenes. Métodos. Un total de 152 deportistas (media de edad: 16,22 \pm 0,80 años) participaron voluntariamente en este estudio. La morfología sagital de las curvas torácica y lumbar, así como la inclinación pélvica, fueron evaluadas con un Spinal Mouse al realizar los test sit-and-reach, toe-touch y McRae \& Wright. La extensibilidad isquiosural fue determinada mediante el test de elevación de la pierna recta (EPR). En base al valor obtenido en el test EPR, la muestra fue dividida en tres grupos

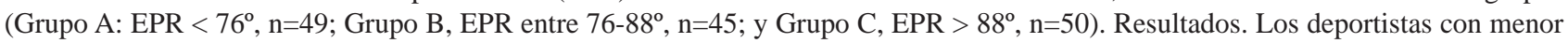
extensibilidad isquiosural adoptaron posturas de mayor cifosis torácica y una mayor retroversión pélvica en los test sit-and-reach y toetouch. No obstante, no se encontraron diferencias significativas entre grupos en el test McRae \& Wright. Además, la extensibilidad isquiosural no tuvo influencia alguna en la morfología sagital del raquis lumbar. Conclusiones. Una menor extensibilidad isquiosural está relacionada con una mayor cifosis torácica y una mayor retroversión pélvica cuando se realizan movimientos de flexión máxima del tronco con rodillas extendidas. La extensibilidad isquiosural no afecta a la disposición sagital del raquis lumbar en movimientos de flexión máxima del tronco.

PALABRAS CLAVE: Postura; Curvaturas sagitales; Columna vertebral; Pelvis; Deporte.

\section{INTRODUCCIÓN}

Los músculos isquiosurales constituyen un grupo muscular biarticular con acciones directas o indirectas sobre las articulaciones de la rodilla y de la cadera y sobre la estática y dinámica lumbo-pélvica. Debido a su origen anatómico en la tuberosidad isquiática, su extensibilidad puede tener cierta influencia en movimientos de flexión del tronco. Una adecuada extensibilidad isquiosural es considerada un importante componente de la condición física saludable y un factor implicado en la salud del raquis. La disminución de la extensibilidad isquiosural se ha relacionado con diversas repercusiones raquídeas (Biering-Sorensen, 1984; Standaert \& Herring, 2000), lesiones musculares (Cabry \& Shiple, 2000) y alteraciones en el ritmo lumbo-pélvico (Esola et al., 1996). Ferrer (1998) estableció una relación directa entre una extensibilidad isquiosural reducida y determinadas repercusiones en el raquis tóraco-lumbar.
Por todo ello, diversos estudios han comparado la postura del raquis y pelvis entre sujetos con diferente extensibilidad isquiosural. Gajdosik et al. (1994) así como Tully \& Stillman (1997) mostraron, en adultos jóvenes, un rango diferente de flexión raquídea en función de la extensibilidad isquiosural. Congdon et al. (2005) encontraron diferencias significativas en la posición de la pelvis entre sujetos con baja y alta extensibilidad al realizar el test de elevación de la pierna recta. Carregaro \& Coury (2009) encontraron que los sujetos con reducida extensibilidad isquiosural presentaban una mayor flexión del tronco y una restricción en el movimiento pélvico al manejar cargas. López-Miñarro \& Alacid (2010) encontraron diferencias en la disposición sagital del raquis en piragüistas en función de su grado de extensibilidad isquiosural en el test sit-and-reach. Sahrmann (2002) indicó que al realizar movimientos de

\footnotetext{
Facultad de Educación. Universidad de Almería, Almería, España.

** Facultad de Ciencias del Deporte. Universidad de Murcia, Murcia, España.

**** Facultad de Educación. Universidad de Murcia, Murcia, España.

Este estudio ha sido financiado con una ayuda de la Fundación Séneca-Agencia de Ciencia y Tecnología de la Región de Murcia (II PCTRM 20072010) con $n^{\circ}$ 11951/PI/09 (Evolución de las curvaturas sagitales del raquis, extensibilidad isquiosural, dolor lumbar y características antropométricas de piragüistas de élite).
} 
flexión máxima del tronco, aquellos sujetos con una reducida extensibilidad presentaban un mayor rango de movimiento intervertebral.

Las posturas de mayor flexión intervertebral se han asociado con un aumento de la presión intradiscal y un riesgo más elevado de repercusiones raquídeas en las estructuras discales, óseas y ligamentosas al aumentar las cargas compresivas, de cizalla anterior y la presión intradiscal (Briggs et al., 2007; Polga et al., 2004; Wilke et al., 1999).

La mayoría de estudios que han analizado la influencia de la extensibilidad isquiosural en la morfología raquídea y pelvis se han llevado a cabo en población no deportista. Puesto que diversos estudios han encontrado adaptaciones raquídeas relacionadas con las posturas y posiciones adoptadas durante los entrenamientos (LópezMiñarro et al., 2009a, 2010a, 2010b; Muyor et al., 2011a, 2011b), el objetivo del presente estudio fue determinar la disposición sagital del raquis e inclinación pélvica en diferentes posiciones, en función del grado de extensibilidad isquiosural en deportistas.

\section{MATERIAL Y MÉTODO}

Participantes. Un total de 152 deportistas (media de edad: $16,22 \pm 0,8$ años) participaron voluntariamente en este estudio. Los criterios de exclusión fueron: 1) haber manifestado dolor lumbar en los tres meses anteriores a la realización del estudio; 2) haber sido operado de la columna vertebral o miembros inferiores; y 3) tener dolor lumbar o torácico en el momento de las valoraciones.

Procedimientos. El estudio fue aprobado por el Comité Ético y de Investigación de la Universidad de Murcia. Previamente a las mediciones, todos los sujetos y sus tutores legales, fueron informados sobre los procedimientos del estudio y firmaron, voluntariamente, un consentimiento informado.

Previamente a las mediciones, se identificó mediante palpación y se marcó, con un lápiz dérmico, el proceso espinoso de la séptima vértebra torácica (C7), así como la tercera vértebra sacra (S3). A continuación, la disposición sagital de la curva torácica y lumbar, así como el ángulo de inclinación pélvica fueron valoradas mediante un Spinal Mouse (Idiag, Suiza), en un orden aleatorio, en los test sitand-reach, toe-touch y MacRae \& Wright. Entre cada medición hubo 5 minutos de descanso. Cada sujeto fue evaluado por el mismo examinador en una misma sesión de valoración. La temperatura del laboratorio donde se realizaron las mediciones fue estandarizada a $24^{\circ} \mathrm{C}$.
Para medir la morfología raquídea, una vez que el sujeto se colocaba en la posición a medir, se situaba el Spinal Mouse en la marca de C7 y se desplazaba en sentido cráneo-caudal a lo largo de los procesos espinosos de las vértebras, hasta la marca de S3. A continuación, el software del sistema digitalizaba el contorno del raquis en el plano sagital, aportando información sobre la angulación global de las curvas raquídeas e inclinación pélvica.

\section{Posturas}

Test sit-and-reach y toe-touch. Para realizar el test, el deportista se situó en sedentación (sit-and-reach) o en bipedestación (toe-touch), con las rodillas extendidas y los pies separados a la anchura de sus caderas. Las plantas de los pies se colocaron perpendiculares al suelo, en contacto con el cajón de medición. En esta posición el deportista realizó una flexión máxima del tronco con rodillas y codos extendidos. Las palmas de las manos, una sobre la otra, se tenían que deslizar sobre el cajón, hasta alcanzar la máxima distancia posible, manteniendo la posición durante 5 segundos. Al alcanzar la máxima distancia, se procedía a la medición de la morfología del raquis mediante el Spinal Mouse.

Test MacRae \& Wright. Para realizar el test, el deportista se colocaba sentado en un banco, colocando los muslos en una posición horizontal, las rodillas en un ángulo de $90^{\circ}$, y las plantas de los pies apoyadas en el suelo. Manteniendo en todo momento el contacto de las plantas de los pies en la superficie de apoyo, el sujeto realizaba una flexión máxima del tronco.

Extensibilidad isquiosural. Para determinar la extensibilidad isquiosural de los deportistas se utilizó el test de elevación de la pierna recta (EPR). Con el deportista en decúbito supino sobre una camilla, con un Lumbosant colocado bajo el raquis lumbar y la pelvis, se procedió a la elevación de la pierna, manteniendo la rodilla extendida, de forma lenta y progresiva hasta que el explorado manifestó dolor o malestar y/o se detectó una retroversión pélvica. Para determinar el ángulo de flexión coxofemoral se colocó un inclinómetro Unilevel (ISOMED, Inc., Portland, OR) en la tuberosidad tibial, colocándolo a cero grados en la posición inicial y estableciendo los grados de flexión coxofemoral al finalizar la misma. Las consignas que se aportaron a los sujetos fueron: "Vamos a elevar la pierna poco a poco. Tienes que dejarla totalmente relajada y has de soportar el estiramiento todo lo que puedas hasta que la tensión te provoque dolor, momento en el que debes avisarnos, diciendo ¡Ya!”. La medición se realizó en ambas piernas por separado y de forma aleatoria. Un investigador mantuvo la pierna contralateral extendida y en contacto con la camilla, evitando la rotación lateral, así como la rotación de la pelvis en su 
eje longitudinal. Otro se encargó de fijar y controlar la basculación de la pelvis.

Análisis estadístico. Una vez obtenidos los datos de los diferentes test, y previamente al análisis estadístico, la población ( $\mathrm{n}=152)$ fue dividida en tres grupos. El criterio para determinar a qué grupo pertenecía cada participante fue el valor angular promedio del test EPR (ángulo miembro inferior izquierdo más ángulo del miembro inferior derecho, dividido entre dos). Aquellos deportistas que presentaban una diferencia mayor de $10^{\circ}$ entre los valores del EPR de ambos miembros fueron excluidos del análisis $(n=8)$. A continuación, fueron asignados al grupo de extensibilidad isquiosural baja (grupo A) si el valor del test EPR era menor de $76^{\circ}(n=49)$, al grupo de extensibilidad isquiosural moderada (grupo B) si el valor estaba entre 76 y $88^{\circ}(n=45)$, o al grupo de extensibilidad isquiosural alta $(n=50) \mathrm{si}$ el valor era mayor de $88^{\circ}$. Los valores límites seleccionados se basaron en la obtención de una distribución lo más homogénea posible de los participantes entre los tres grupos.

A continuación se realizó un análisis descriptivo de cada una de las variables dependientes, obteniendo sus valores medios y desviaciones típicas. Tras aplicar el test de normalidad de Kolmogorov-Smirnov y comprobar que las variables seguían una distribución normal, se realizó un análisis de varianza (ANOVA) de un factor para comparar la morfología raquídea en función del grado de extensibilidad isquiosural. Si se encontraban diferencias significativas en las variables dependientes para el efecto principal del ANOVA ( $\mathrm{p}<0,05)$, se procedió a realizar una comparación por pares (post hoc) usando la corrección de Bonferroni para comparaciones múltiples, ajustando el criterio de significación a un valor de 0,016 (0,05 dividido entre 3). Los datos fueron analizados usando el software SPSS (versión 15,0), y el nivel de significación se estableció en un valor de $\mathrm{p}<0,05$.

\section{RESULTADOS}

Los valores medios ( \pm desviación típica) del test de elevación de la pierna recta fueron de $69,77 \pm 6,80^{\circ}, 83,42 \pm 5,93^{\circ}$ y $94,20 \pm 7,17^{\circ}(\mathrm{p}<0,001)$ para la pierna derecha en los grupos A, B y C, respectivamente. En la pierna izquierda, los valores fueron de $71,04 \pm 7,50^{\circ}, 83,81 \pm 8,49^{\circ}$ y $92,61 \pm 11,00^{\circ}$, respectivamente $(\mathrm{p}<0,001)$.

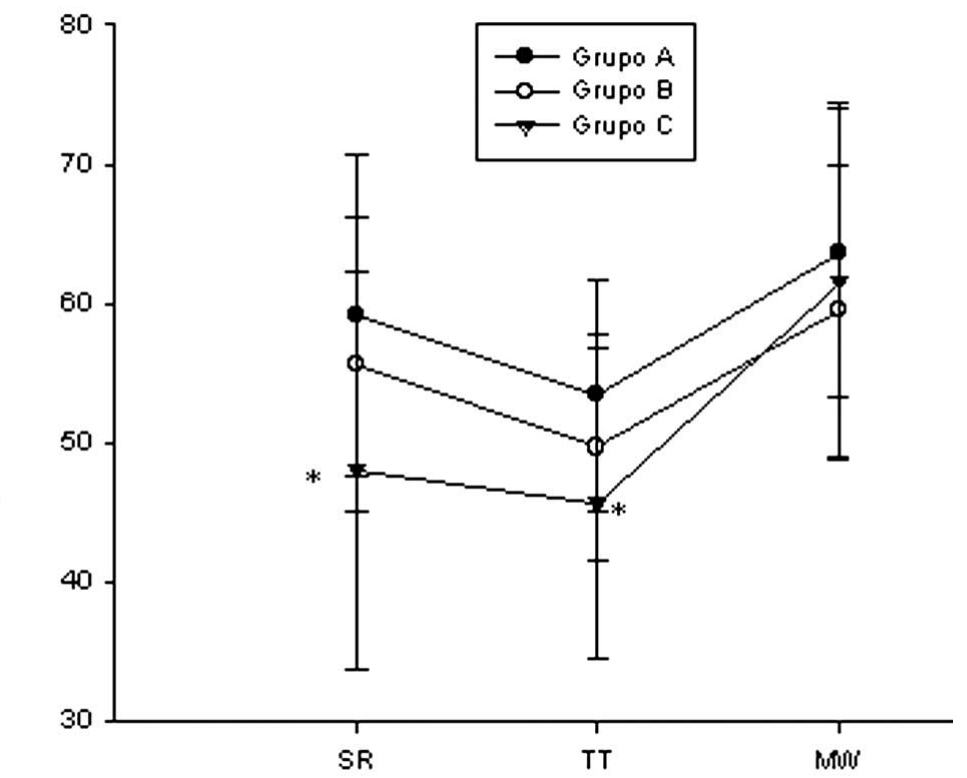

Fig. 1. Media \pm desviación estándar de la curva torácica en los test de flexión del tronco. SR: test sit-and-reach; TT: test toe-touch; MW: test MacRae \& Wright; $* \mathrm{p}<0,016$ respecto al grupo A.

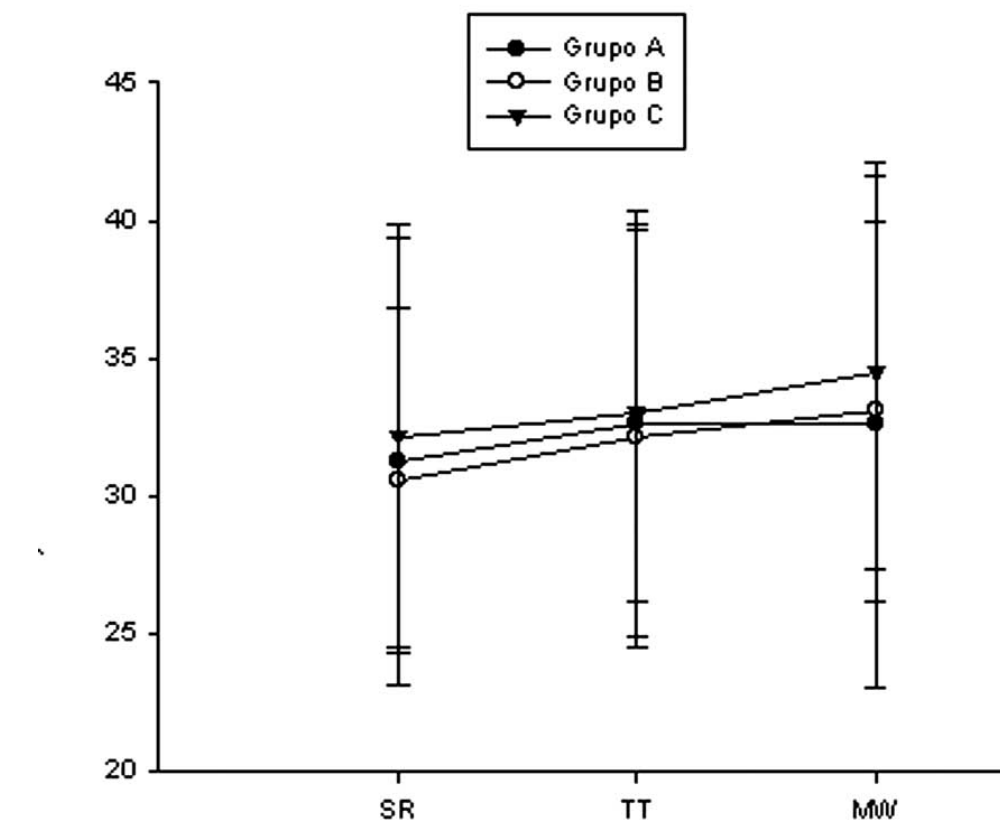

Fig. 2. Media \pm desviación estándar de la curva lumbar en los test de flexión del tronco. SR: test sit-and-reach; TT: test toe-touch; MW: test MacRae \& Wright. 


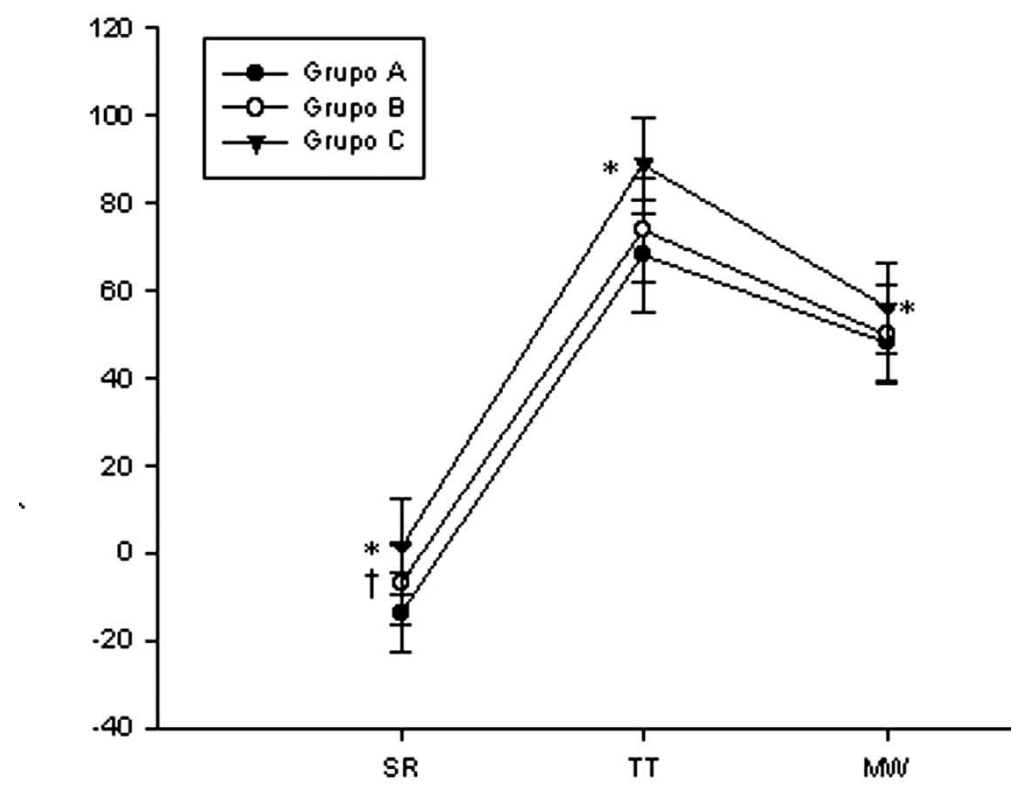

Fig. 3. Media \pm desviación estándar de la inclinación pélvica en los test de flexión del tronco. SR: test sit-and-reach; TT: test toe-touch; MW: test MacRae \& Wright; * $\mathrm{p}<0,016$ respecto al grupo A; $\uparrow \mathrm{p}<0,016$ respecto al grupo A.

Los valores medios ( \pm desviación típica) de las curvas torácica y lumbar, e inclinación pélvica en las posiciones evaluadas se presentan en las Figuras 1, 2, y 3, respectivamente. El ANOVA mostró diferencias significativas para el efecto principal de la curva torácica en los test sit-and-reach $(\mathrm{F}=10,515$; $\mathrm{p}<0,001)$ y toetouch $(\mathrm{F}=8,775 ; \mathrm{p}<0,001)$, pero no en el MacRae \& Wright. Sin embargo, en la curva lumbar el ANOVA evidenció que no existían diferencias significativas.

Respecto a la pelvis, se encontraron diferencias significativas en el efecto principal del ANOVA en los tres test: sit-and-reach $(\mathrm{F}=24,237 ; \mathrm{p}<0,001)$, toe-touch $(\mathrm{F}=30,979 ; \mathrm{p}<0,001)$ y MacRae $\&$ Wright $(\mathrm{F}=7,591 ; \mathrm{p}<0,001)$. Tanto en el raquis torácico como en la inclinación pélvica el análisis post hoc evidenció diferencias significativas entre los grupos de extensibilidad isquiosural baja (Grupo A) y alta (Grupo C).

\section{DISCUSIÓN}

El objetivo principal del estudio fue determinar la influencia del grado de extensibilidad isquiosural en la disposición sagital del raquis e inclinación pélvica en deportistas. Los resultados principales evidencian una relación entre el grado de extensibilidad isquiosural y la posición del raquis torácico e inclinación pélvica en las posiciones de flexión máxima del tronco con rodillas extendidas. Estos datos coinciden con Sahrmann, que refiere un mayor rango de movimiento intervertebral al realizar movimientos de flexión máxima del tronco en sujetos con una reducida extensibilidad isquiosural. Sin embargo, cuando se realiza una flexión máxima del tronco manteniendo las rodillas flexionadas a $90^{\circ}$, desaparece dicha relación, ya que se produce una disminución de la tensión muscular en los músculos isquiosurales, permitiendo que la pelvis pueda girar más en sentido anterior, de forma muy significativa, respecto a las posiciones con rodillas extendidas. Estudios previos han encontrado que la extensibilidad isquiosural no tiene influencia en la disposición sagital del raquis en las posiciones de bipedestación o sedentación relajada, en las que los músculos isquiosurales tienen poca tensión (Gajdosik et al.; López-Miñarro \& Alacid).

En adultos jóvenes y deportistas se ha mostrado que la extensibilidad isquiosural condiciona la disposición angular del raquis y la pelvis en máxima flexión del tronco con rodillas extendidas, de modo que las personas con mayor extensibilidad presentan una mayor flexión pélvica, así como menor flexión torácica (Gajdosik et al.; Rodríguez-García et al., 2008; Tully \& Stillman). En el presente estudio, los deportistas con mayor extensibilidad isquiosural adoptaron posturas de menor cifosis torácica en las posiciones de flexión máxima del tronco. Estos datos coinciden con estudios previos en deportistas jóvenes (López-Miñarro \& Alacid) y en adultos sedentarios (Gajdosik et al.). Este hecho está probablemente relacionado con la mayor flexión pélvica alcanzada por los deportistas con mayor grado de extensibilidad. De este modo, al quedar el tronco más inclinado en sentido anterior, debido a una mayor inclinación pélvica en sentido anterior, no es preciso forzar el movimiento de flexión torácica para alcanzar mayor distancia (López-Miñarro et al., 2009b). Respecto a la movilidad pélvica, estudios realizados en adultos no deportistas refieren diferencias significativas en la posición de la pelvis entre sujetos con baja y alta extensibilidad (Congdon et al.; Carregaro \& Coury).

No obstante, el raquis lumbar, a pesar de ser una estructura vertebral directamente relacionada con la posición de la pelvis (Levine \& Whittle, 1996), no está influido por la extensibilidad isquiosural. Estos datos coinciden con estudios previos que encuentran bajos valores de correlación entre la extensibilidad isquiosural y la posición del raquis lumbar en movimientos de flexión máxima del tronco con rodillas extendidas (López-Miñarro et al., 2009b; RodríguezGarcía et al.). Resulta destacable que aunque no haya 
una relación estadísticamente significativa, las personas con mayor extensibilidad isquiosural alcanzan mayores valores de flexión lumbar que los sujetos con una reducida extensibilidad. Esta relación ha sido también referida en otros estudios (López-Miñarro et al., 2009a, 2010a).

Las posturas de mayor flexión intervertebral están asociadas a mayores cargas compresivas y de cizalla en las estructuras intervertebrales (Briggs et al.; Polga et al.; Wilke et al.; Keller et al., 2005). Además, la adopción de posturas cifóticas mantenidas o repetitivas en un raquis aún en proceso de maduración puede producir alteraciones en los núcleos de crecimiento de las vértebras (Wojtys et al., 2000). Pastor (2000) encontró mayor presencia de acuñamientos vertebrales en la transición tóraco-lumbar en nadadores que presentaban mayor flexión intervertebral torácica en el test sit-and-reach. La edad de los deportistas evaluados en este estudio supone que sus núcleos de crecimiento vertebral aún siguen activos y, por tanto, una postura inadecuada del raquis podría generar alteraciones que afectarían a su calidad de vida, así como a su trayectoria deportiva.

En la mayoría de ocasiones, una reducida extensibilidad isquiosural es un problema asociado a la ausencia de un trabajo continuado de esta capacidad porque los técnicos deportivos, y los propios deportistas, no consideran la extensibilidad isquiosural como una capacidad importante en la consecución de un alto rendimiento deportivo (Nyland et al., 2004). Sin embargo, una reducida extensibilidad tiene influencia sobre la disposición sagital del raquis torácico y pelvis, de modo que a menor extensibilidad se adoptan posiciones de mayor flexión torácica y retroversión pélvica. En este sentido, Ferrer y Pastor, en deportistas jóvenes, encontraron una asociación significativa entre una reducida extensibilidad isquiosural y el porcentaje de repercusiones en el raquis lumbar y la charnela tóraco-lumbar. Por ello, se debería incrementar el correcto trabajo de extensibilidad de la musculatura isquiosural en los entrenamientos.

En conclusión, una menor extensibilidad isquiosural en deportistas está relacionada con una mayor cifosis torácica y una mayor retroversión pélvica cuando se realizan movimientos de flexión máxima del tronco con rodillas extendidas. La extensibilidad isquiosural no afecta a la morfología sagital del raquis lumbar en movimientos de flexión del tronco.

AGRADECIMIENTOS. Los autores agradecen su colaboración a los entrenadores y deportistas que participaron en el estudio.

MUYOR, J. M.; ALACID, F.; RODRÍGUEZ-GARCÍA, P. L. \& LÓPEZ-MIÑARRO, P. A. Influence of hamstring extensibility on sagittal spinal curvatures and pelvic inclination in athletes. Int. J. Morphol., 30(1):176-181, 2012.

SUMMARY: The objective of this study was to determine the influence of hamstring extensibility in sagittal spinal curvatures and pelvic inclination on young athletes. Methods: A hundred and fifty-two young athletes (mean age: $16.22 \pm 0.80$ years) were recruited. Thoracic and lumbar curvatures and pelvic inclination were evaluated with a Spinal Mouse system in the sit-and-reach test, toe-touch test and McRae \& Wright test. Hamstring muscle extensibility was determined by passive straight leg raise test (PSLR). The sample was divided into three groups with regard to straight leg raise angle (Group A: PSLR $<76^{\circ}, n=49$; Group B, PSLR between 76-88 ${ }^{\circ}$, $=45$; and Group C, PSLR > 88, $\mathrm{n}=50$ ). Results. Athletes with lower extensibility presented higher thoracic angle and a more posterior pelvic tilt in the sit-and-reach and toe-touch tests. However, no significant differences were found between groups when maximal trunk flexion with knees flexed was performed (McRae \& Wright test). The lumbar curve was not affected by hamstring extensibility in any position. Conclusions: Lower hamstring extensibility is related to increased thoracic curve and more posterior pelvic tilt when maximal trunk flexion with knees extended is performed. Hamstring extensibility has not any influence in sagittal lumbar morphology when trunk flexion is performed.

KEY WORDS: Posture; Sagittal curvatures; Spine; Pelvis; Sports.

\section{REFERENCIAS BIBLIOGRÁFICAS}

Biering-Sorensen, F. Physical measurements as risk indicator for low-back trouble over a one year period. Spine, 9:10619, 1984.

Briggs, A. M.; van Dieën, J. H.; Wrigley, T. V.; Greig, A. M.; Phillips, B.; Lo, S. \& Bennell, K. L. Thoracic kyphosis affects spinal loads and trunk muscle force. Phys. Ther., 87:595-607, 2007.
Cabry, J. \& Shiple, B. J. Increasing hamstring flexibility decreases hamstring injuries in high school athletes. Clin. J. Sport Med., 10:311-2, 2000.

Carregaro, R. L. \& Coury, H. J. C. Does reduced hamstring flexibility affect trunk and pelvic movement strategies during manual handling? Int. J. Ind. Ergon., 39:115-20, 2009. 
Congdon, R.; Bohannon, R. \& Tiberio, D. Intrinsic and imposed hamstring length influence posterior pelvic rotation during hip flexion. Clin. Biomech., 20:947-51, 2005.

Esola, M. A.; McClure, P. W.; Fitzgerald, G. K. \& Siegler, S. Analysis of lumbar spine and hip motion during forward bending in subjects with and without a history of low back pain. Spine, 21:71-8, 1996.

Ferrer, V. Repercusiones de la cortedad isquiosural sobre la pelvis y el raquis lumbar. Tesis Doctoral, Murcia, Universidad de Murcia, 1998.

Gajdosik, R. L.; Albert, C. R. \& Mitman, J. J. Influence of hamstring length on the standing position and flexion range of motion of the pelvic angle, lumbar angle, and thoracic angle. J. Orthop. Sports Phys. Ther, 20:213-9, 1994.

Keller, T. S.; Colloca, C. J.; Harrison, D. E.; Harrison, D. D. \& Janik, T. J. Influence of spine morphology on intervertebral disc loads and stresses in asymptomatic adults: implications for the ideal spine. Spine J., 5:297309, 2005.

Levine, D. \& Whittle, M. W. The effects of pelvic movement on lumbar lordosis in the standing position. J. Orthop. Sports Phys. Ther., 24:130-5, 1996.

López-Miñarro, P. A. \& Alacid, F. Influence of hamstring muscle extensibility on spinal curvatures in young athletes. Sci. Sports., 25:88-93, 2010.

López-Miñarro, P. A.; Alacid, F. \& Muyor, J. M. Comparación del morfotipo raquídeo y extensibilidad isquiosural entre piragüistas y corredores. Rev. Int. Med. Cienc. Ac., 9:379-92, 2009a.

López-Miñarro, P. A.; Sáinz de Baranda, P. \& RodríguezGarcía, P. L. A comparison of the sit-and-reach test and the back-saver sit-and-reach test in university students. $J$. Sports Sci. Med., 8:116-22, 2009b.

López-Miñarro, P. A.; Alacid, F. \& Rodríguez-García, P. L. Comparison of sagittal spinal curvatures and hamstring muscle extensibility among young elite paddlers and nonathletes. Int. Sport Med. J., 11:301-12, 2010a.

López-Miñarro, P. A.; Muyor, J. M. \& Alacid, F. Sagittal spinal curvatures and pelvic tilt in elite young kayakers. Med. Sport, 63:509-19, 2010b.

Muyor, J. M.; López-Miñarro, P. A. \& Alacid, F. Spinal posture of thoracic and lumbar spine and pelvic tilt in highly trained cyclists. J. Sports Sci. Med., 10:355-61, 2011a.

Muyor, J. M.; López-Miñarro, P. A. \& Alacid, F. Sagittal spinal morphology in young highly-trained paddlers. Int. J. Morphol., 29:1047-53, 2011b.

Nyland, J.; Kocabey, Y. \& Caborn, D. N. Sex differences in perceived importance of hamstring stretching among high school athletes. Percept. Mot. Skills, 99:3-11, 2004.

Pastor A. Estudio del morfotipo sagital de la columna y de la extensibilidad de la musculatura isquiosural de jóvenes nadadores de élite Españoles. Tesis Doctoral, Murcia, Universidad de Murcia, 2000.

Polga, D. J.; Beaubien, B. P.; Kallemeier, P. M.; Schellhas, K. P.; Lee, W. D.; Buttermann, G. R. \& Wood, K. B. Measurement of in vivo intradiscal pressure in healthy thoracic intervertebral discs. Spine, 29:1320-4, 2004.

Rodríguez-García, P. L.; López-Miñarro, P. A.; Yuste, J. L. \& Sáinz de Baranda, P. Comparison of hamstring criterionrelated validity, sagittal spinal curvatures, pelvic tilt, and score between sit-and-reach and toe-touch tests in athletes. Med. Sport, 61:11-20, 2008.

Sahrmann, S. A. Diagnosis and treatment of movement impairment syndromes. St. Louis, Mosby, 2002.

Standaert, C. J. \& Herring, S. A. Spondylolysis: A critical review. Br. J. Sports Med., 34:415-22, 2000.

Tully, E. A. \& Stillman, B. C. Computer-aided video analysis of vertebrofemoral motion during toe touching in healthy subjects. Arch. Phys. Med. Rehabil., 78:759-66, 1997.

Wilke, H. J.; Neef, P.; Caimi, M.; Hoogland, T. \& Claes, L. E. New in vivo measurements of pressures in the intervertebral disc in daily life. Spine, 24:755-62, 1999.

Wojtys, E. M.; Ashton-Miller, J. A.; Huston, L. J. \& Moga, P. J. The association between athletic training time and sagittal curvature of the immature spine. Am. J. Sports Med., 28:490-8, 2000.

Dirección para correspondencia:

Pedro A. López-Miñarro.

Department of Physical Education.

Faculty of Education, Campus Universitario de Espinardo CP. 30100 Murcia

ESPAÑA

Email: palopez@um.es

Recibido : 07-07-2011

Aceptado: 24-10-2011 